\title{
Qualitative Evidence Synthesis on Self-Collection for Human Papillomavirus-Based Cervical Screening: Protocol for Systematic Review
}

Hawa Camara ${ }^{1}$, BS, MPH, CHES; Ye Zhang ${ }^{1}$, BS, MPH; Lise Lafferty ${ }^{1,2}$, BA, MA, MA, PhD; Andrew Vallely ${ }^{1,3}$, MBBS, MRCP, DTMH, MSc, DLSHTM, PhD; Rebecca Guy ${ }^{1}$, MAppEpid, PhD; Angela Kelly-Hanku ${ }^{1,3}$, BA (Hon), $\mathrm{PhD}$

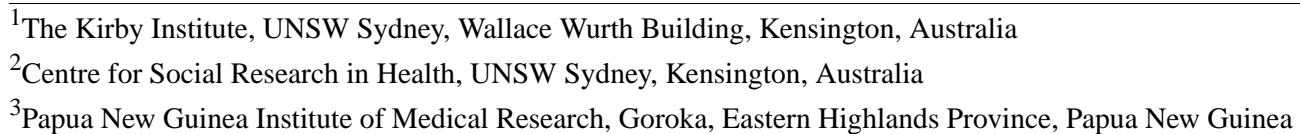

\section{Corresponding Author:}

Hawa Camara, BS, MPH, CHES

The Kirby Institute

UNSW Sydney

Wallace Wurth Building

Kensington

Australia

Phone: 610478118162

Email: camhaw@gmail.com

\section{Abstract}

Background: Cervical cancer is the fourth most common cancer affecting women worldwide. In the 1980s, it was found that the sexually transmitted disease human papillomavirus causes over $90 \%$ of all cervical cancer cases. Since that discovery, diagnostic technologies have been developed for the detection of human papillomavirus DNA in cervical samples. However, significant sociocultural and structural barriers remain. Considerable strides have taken place in recent years to address these barriers, such as the self-collection for human papillomavirus-based cervical screening method.

Objective: The purpose of this review is to synthesize qualitative evidence around the self-collection method and identify strategies to increase acceptability and feasibility in different settings. This qualitative synthesis will be used to better understand how to conceptualize and implement more effective, accessible, and socially and culturally acceptable cervical screening programs and policies globally.

Methods: A systematic search will be conducted in Global Health, Cochrane, CINAHL (Cumulative Index to Nursing and Allied Health Literature), ProQuest, ScienceDirect, EMBASE, EMCARE, Medline (OVID), Scopus, and Web of Science. Published and peer-reviewed articles will be included. Two reviewers will independently screen and assess the studies. The data will be coded and analyzed using a thematic synthesis process. The socioecological model will be used to organize emergent themes at the micro and macro levels. The results will be presented in narrative and tabular form.

Results: The article search and data extraction were completed in May 2020. The data were analyzed in June 2020. The review will be submitted for publication in Fall 2020.

Conclusions: This review will present the global evidence of the perspectives and experiences of various key stakeholders and how these perspectives and experiences impact their decision-making process to perform or accept self-collection for human papillomavirus-based cervical screening. The review will provide guidance to implementation researchers as well as implications for future research.

Trial Registration: PROSPERO International Prospective Register of Systematic Reviews CRD42019109073; https://www.crd.york.ac.uk/PROSPERO/display_record.php?RecordID=109073

International Registered Report Identifier (IRRID): DERR1-10.2196/21093

(JMIR Res Protoc 2020;9(10):e21093) doi: 10.2196/21093 


\section{KEYWORDS}

self-collection; HPV-based testing; cervical screening; qualitative evidence synthesis; protocol; systematic review

\section{Introduction}

Cervical cancer is the fourth leading cause of cancer affecting women in the world, with the highest burden of disease, estimated at $85 \%$ [1], occurring in low- and middle-income countries. To attenuate the global cervical cancer burden, the implementation of screening methods is a global health priority. The most commonly known screening method is cytologic screening with a Papanicolaou test (Pap smear): since its introduction in the $1940 \mathrm{~s}$, cervical cancer incidence and mortality have decreased by $70 \%$ in high-income countries [2]. However, Pap smears require distinctive and extensive laboratory infrastructure and capacity, making it challenging to implement in resource-limited settings $[3,4]$.

In the early 1980s, researchers discovered the presence of human papillomavirus (HPV) DNA in cervical cancer lesions and that the sexually transmitted infection (STI) was responsible for over $90 \%$ of malignant cases [5]. Subsequent research has demonstrated that persistent HPV infection puts women at higher risk for cervical cancer [6]. These research findings have led to the development of tests for the detection of oncogenic HPV types in cervical precancerous or cancerous lesions, referred to as HPV testing. Additionally, this was an opportunity to develop technologies that are low-cost and require minimal laboratory infrastructure and training to use. Since this discovery of HPV oncogenic types, technologies for conducting HPV testing have revolutionized the way the global health community views cervical screening and its impact on global health outcomes [7]. The new screening methods offer access to screening services at a relatively low-cost via an effective and efficient process that bypasses all laboratory needs required by the Pap smear. Most of the research to date has been focused on determining the effectiveness of HPV-based cervical screening methods. A global review by Kouliopoulos et al [8] of studies conducted between 1992 and 2015 showed that HPV testing has higher sensitivity and specificity than those of conventional cytology methods and allows for less frequent screening. When provided at point-of-care, patients receive their results in a shorter time frame (60-90 minutes on average, compared to up to 2 weeks for Pap smears) allowing for faster turnaround, more timely treatment, and reduction in patients lost to follow-up $[4,9,10]$. Research from Goldie et al [11] demonstrated that HPV-based screening for women once they have reached 35 years of age potentially reduces the lifetime risk of cervical cancer by $36 \%$.

The concept of self-collection (interchangeably named self-collected samples or self-sampling) was introduced in the 1970 s to address the alarmingly low rates among women who lacked access to screening and health care services, often due to sociocultural, economic, and structural factors [12]. In the 1990s, Dr. Arthur Fournier noticed the concerning underutilization rates of Pap smear screening in Haiti. To circumvent the discomfort and embarrassment of pelvic examinations, a known deterrent for women seeking cervical screening services, Dr. Fournier designed a cervical self-sampling device for the detection of cancer and STIs [13]. The innovative self-sampling device that could also be used at home provided an efficient solution to screen women in resource-limited and culturally challenging settings. Since then, numerous studies [14-17] have compared the efficacy and effectiveness of self-collected samples to clinician-collected samples for cervical screening. The evidence showed that self-collected sampling not only had comparable sensitivity and specificity to those of clinician-collected sampling but was also the most-widely preferred screening method by women. Other research [18] has demonstrated that self-collection of specimens for HPV testing could potentially increase uptake for women residing in hard-to-reach settings.

With the vast and rapid progress in cervical screening methods, it is essential to recognize that, when applied in varying contexts and among different people, responses and experiences will significantly differ. In the case of self-collection for HPV screening, social values, cultural values, and understanding of the female body can each have an impact on women's acceptability of innovative cervical screening methods. To be successful, methods such as self-collection, need to be socially and culturally accepted by women, health care workers, and policymakers [19], as well as fit within settings where access to screening is scarce due to sociocultural barriers and limited resources. This requires understanding the experiences of all key actors (ie, women, health care workers, and policymakers) at all levels of the health system interacting with it.

This review aims to generate findings for further guidance to implementation researchers. It will help with the design of self-collection for HPV-based cervical screening interventions that address all factors raised by key stakeholders that could impact the acceptability and feasibility of these programs in diverse cultural and geographical settings.

To facilitate the uptake of cervical screening, thus reducing the incidence and prevalence of cervical cancer globally, this systematic review aims to identify, analyze, and synthesize the experiences and perceptions of women, health care workers, and policymakers. Consequently, it will provide evidence of factors that impact the acceptability and feasibility of self-collection for HPV-based cervical screening globally.

\section{Methods}

\section{General}

A preliminary search for existing systematic reviews on the topic has been conducted in major databases (ie, Medline; Scopus; Joanna Briggs Institute Database of Systematic Reviews and Implementation Reports; Cochrane Database; CINAHL, Cumulative Index to Nursing and Allied Health Literature; PubMed; and PROSPERO, International Prospective Register of Systematic Reviews). We found no qualitative synthesis systematic reviews that explored factors that could impact the acceptability and feasibility of self-collection for HPV-based cervical screening from the perspectives of women, health care 
workers, and policymakers, globally. The protocol will follow PRISMA (Preferred Reporting Items for Systematic Reviews and Meta-Analyses) guidelines [20]. Participant, interest, and context inclusion criteria [21] used for this review are detailed.

\section{Review Questions}

What are women's, health care workers', and policymakers' experiences with and perceptions of self-collection for HPV-DNA cervical cancer screening?

What are the barriers and facilitators to the acceptability and implementation of self-collection for HPV-DNA cervical cancer screening from the point of view of women, health care workers, and policymakers?

\section{Types of Participants}

The review will focus on women, health care workers, and policymakers. For women participants, there will be no age restriction since the review will focus on both perspectives and experiences of the self-collection method, albeit the HPV-DNA test is recommended for women 30 years old and above only. Also, the reviewers will allow for different terms to be used when identifying the types of participants targeted in this review: for example, health care workers could be defined differently in studies (ie, providers, health care service providers, etc).

\section{Phenomena of Interest}

The intervention of interest in this review is self-collection for HPV-based cervical screening. This review will consider all elements associated with the process of self-collection. The first outcome will be to assess the sociocultural and structural barriers and facilitators from the point of view of women (ie, patients), health care workers, and policymakers as well as to determine the feasibility factors of self-collection for HPV-based cervical screening in different settings.

\section{Context}

The review will not have a global geographical restriction: all countries with publicly available data will be included. Additionally, any setting where self-collection for HPV-based cervical screening could be performed (which includes women's homes, primary care, community health center) will also be identified and included in the review.

\section{Study Search Methods}

\section{Types of Studies}

This review will consider qualitative and mixed methods studies (Textbox 1) that draw on the experiences and perspectives of women, health care workers, and policymakers. These will include designs such as grounded theory, narratives, ethnography, phenomenology, and action research.

Two authors will independently screen the database search results, compare and discuss their findings, and resolve disagreements to reach consensus.

Textbox 1. Criteria.

Inclusion criteria

- Qualitative studies (qualitative component of mixed methods studies, interviews, focus groups, surveys, or questionnaires with open-ended questions) that explored experiences, perspectives of self-sampling or self-collection for human papillomavirus (HPV)-based cervical screening

- Studies that involve women; health care workers including physicians (obstetrics/gynecology primarily), nurses, midwives, and allied health professionals; or policymakers

- $\quad$ Studies published in English

- $\quad$ Studies in any geographical setting (high-income countries and low- and middle-income countries) and health care settings (eg, community health care centers, primary health care centers, patient's homes)

- $\quad$ Peer-reviewed publications published after 1986 (year of the first study on HPV-DNA testing)

\section{Exclusion criteria}

- Studies that involve stakeholders other than the ones listed in the inclusion criteria

- Studies in languages other than English

- Purely quantitative studies and quantitative components of mixed methods studies

- $\quad$ Nonpeer reviewed articles, theses, abstracts, reviews, or book chapters

- $\quad$ Any other cervical screening method that is not self-collection for HPV-based cervical screening (ie, clinician-collected cervical screening, Pap smears, visual inspection with acetic acid or visual inspection with Lugol iodine)

\section{Search Strategy}

A systematic search of the following databases will be developed in collaboration with a librarian and conducted by $\mathrm{HC}$ in Global Health, Cochrane, CINAHL (Cumulative Index to Nursing and Allied Health Literature), ProQuest, ScienceDirect, EMBASE, EMCARE, Medline (OVID), Scopus, and Web of Science. Published peer-reviewed articles will be included.
The literature search will be limited to studies published in English, between 1986 (year of the first study on HPV-DNA testing) to December 2019. It will ensure that both controlled vocabulary, medical subject headings (MeSH) and keywords, are tailored to each database (see an example of the draft EMBASE search strategy and keywords used in Multimedia Appendix 1. 
The search strategy will include terms focused on 4 main concepts: HPV, self-collection, HPV-DNA testing, and qualitative. The primary reviewer $\mathrm{HC}$ will consult with the librarian for help refining the search strategy and terms to ensure the inclusion of both Medical Subject Heading (MeSH) terms and keywords relevant to this review and its aims. A preliminary list of MeSH terms and keywords are listed in Table 1.

Table 1. Terms and keywords.

\begin{tabular}{|c|c|c|}
\hline Concept & Keywords & $\mathrm{MeSH}^{\mathrm{a}}$ \\
\hline \multirow[t]{2}{*}{$\mathrm{HPV}^{\mathrm{b}}$} & Human papillomavirus OR human papilloma virus & $\begin{array}{l}\text { Papillomaviridae OR papillomavirus infections OR } \\
\text { uterine cervical neoplasms }\end{array}$ \\
\hline & AND & AND \\
\hline \multirow[t]{2}{*}{ HPV-DNA testing } & $\begin{array}{l}\text { Cervical cancer screening OR cervical screening OR cervical } \\
\text { ADJ8 screening }\end{array}$ & $\begin{array}{l}H P V-D N A \text { testing OR HPV testing OR } H P V \text { primary } \\
\text { testing OR primary HPV testing OR DNA probes, } H P V \\
\text { OR human papillomavirus DNA tests OR vaginal } \\
\text { smears }\end{array}$ \\
\hline & AND & AND \\
\hline \multirow[t]{2}{*}{ Self-collected } & Self-sampling OR self-collected OR self-administered & $\underline{c}^{\mathrm{c}}$ \\
\hline & AND & AND \\
\hline Qualitative & Qualitative & $\begin{array}{l}\text { Qualitative research } \mathrm{OR} \text { qualitative studies } \mathrm{OR} \text { quali- } \\
\text { tative study } \mathrm{OR} \text { focus groups } \mathrm{OR} \text { interviews as topic } \\
\text { OR observation } \mathrm{OR} \text { ethnography }\end{array}$ \\
\hline
\end{tabular}

${ }^{\mathrm{a}} \mathrm{MeSH}$ : Medical Subject Headings.

${ }^{\mathrm{b}} \mathrm{HPV}$ : human papillomavirus.

${ }^{\mathrm{c}} \mathrm{No} \mathrm{MeSH}$ terms were used.

All articles that are identified will be imported into EndNote (QSR International) to systematically sort, review, and select the final list of articles to be included in the synthesis. Guided by the eligibility criteria, 2 reviewers ( $\mathrm{HC}$ and $\mathrm{YZ}$ ) will screen each article in the Endnote library by title and abstract. The list of articles subjected to full review will be agreed upon between the 2 reviewers ( $\mathrm{HC}$ and $\mathrm{YZ}$ ). The 2 reviewers will proceed to review the full text of all eligible studies. The final list of included studies will be discussed and agreed upon between the 2 reviewers and an additional third reviewer (AKH).

\section{Assessment of Methodological Quality of Included Studies}

All included studies will be critically appraised using the Critical Appraisal Skills Programme tool or CASP for qualitative research [22], albeit there is no clear consensus on a standard tool applied to the methodological appraisal of qualitative studies [23]. This will be conducted independently by 2 reviewers (HC and YZ). The CASP tool consists of 10 questions that assess the essential elements of qualitative research. Each item will be scored using yes (1 point), no (0 points), or "can't tell" ( 0.5 points) to score the article's quality out of 10 .

The quality of the article will be determined using the following scoring guideline: a score $\leq 3$ is of low quality, a score ranging from 4-6 is of medium quality, and a score $\geq 7$ is of high quality. The appraisal score will not be used to exclude articles.

\section{Data Extraction And Analysis}

\section{Data Extraction}

As an initial step, an extraction exercise will be conducted to ensure accuracy, completeness, and richness of the data. An
Excel (Microsoft Inc) data extraction spreadsheet will be developed to include the following study characteristics: title, authors, journal, publication date, study design, research aim, setting and study location, sample size, age group, demographics, intervention or screening type, data collection method, theoretical or conceptual framework, data analysis, outcomes, and findings.

Two reviewers (HC and YZ) will independently extract the data using the data extraction spreadsheet, and any disagreements will be discussed to reach consensus.

\section{Data Synthesis}

Qualitative research findings will be pooled using the thematic synthesis approach. This approach, developed by Thomas and Harden [24], specifically looks at individuals' perspectives and experiences [25] using an integrative approach which considers data from comparable primary studies. For data coding, we will use NVivo (version 12.5; QSR International) qualitative data management and analysis software. Codes will inductively be identified and grouped into themes [26]. The thematic synthesis approach is comprised of 3 stages: coding text where each study will be coded line-by-line extracting data that responds to the research questions developed for this review (HC will conduct this); developing descriptive themes where the codes identified in the first stage will be categorized based on similarities to create themes (HC will conduct this); and generating analytical themes where the themes identified in the second stage will be used to develop key messages (this will be conducted by HC and discussed with and agreed upon by YZ, LL, AV, RG, and AKH). 
As part of the thematic synthesis, a theoretical framework was identified to guide the data synthesis process. For this review, the socioecological model will be the a priori framework that will need to be discussed and approved by all reviewers (HC, YZ, LL, AV, RG, and AKH), used, and adapted to identify emerging themes. Socioecological model views health behavior as being shaped by and influenced at multiple levels [27]: (1) intrapersonal, (2) interpersonal, (3) organizational, (4) community, and (5) public policy. By using the socioecological model, we aim to identify micro and macro factors that impact the perspectives and experiences of using self-collection for HPV testing, thus impacting acceptability from the point of view of all key stakeholders, as well as the implementation of the self-collection method in different settings.

The findings will provide a descriptive summary of key themes and associated quotations.

\section{Patient and Public Involvement}

This review will include solely secondary data, patients, health care workers, policymakers, and the public will not be involved in the design or conduct of the review.

\section{Ethics and Dissemination}

This review will involve the collection and analysis of publicly available secondary data, and therefore, does not require ethical approval. The review findings will be disseminated through publication in a peer-reviewed journal and scientific conference presentations. The review will include relevant discussion points to further guide implementation researchers for the prevention of cervical cancer globally as well as implications for future research.

\section{Results}

The protocol was registered on April 15, 2019 (PROSPERO CRD42019109073). The article search and data extraction were completed in May 2020. The review includes 33 papers published between 2008 and 2020. The data were analyzed in June. The review will be submitted for publication in Fall 2020.

\section{Discussion}

Qualitative research should be a priority very early on in implementation research when introducing socially and structurally sensitive screening programs. This qualitative evidence synthesis will aim to review the perspectives and experiences of key stakeholders and the impact on their decision-making process to perform or accept self-collected HPV-based cervical screening. To date, scoping and systematic reviews on cervical screening have focused on qualitative evidence about Pap smears and visual inspection with acetic acid methods, and most recently, HPV testing. This will be the first review that qualitatively explores the most recent innovative HPV-testing method of self-collection, which is being increasingly used globally. By using the socioecological model, strategies will be identified and discussed to address barriers and facilitators to increase acceptability and feasibility at every level of the system. The review will include relevant discussion points to further guide implementation researchers for the prevention of cervical cancer globally as well as implications for future research.

\section{Acknowledgments}

This project was made possible as part of a research-funded $\mathrm{PhD}$ being undertaken by HC under the University of New South Wales' Scientia scholarship.

\section{Authors' Contributions}

HC drafted the manuscript. HC, YZ, LL, AV, RG, and AKH contributed to the design of the review protocol. All authors read and approved the final manuscript.

\section{Conflicts of Interest}

None declared.

\section{Multimedia Appendix 1 \\ EMBASE draft strategy. \\ [DOCX File, 13 KB-Multimedia Appendix 1]}

\section{References}

1. LaVigne AW, Triedman SA, Randall TC, Trimble EL, Viswanathan AN. Cervical cancer in low and middle income countries: Addressing barriers to radiotherapy delivery. Gynecol Oncol Rep 2017 Nov;22:16-20 [FREE Full text] [doi: 10.1016/j.gore.2017.08.004] [Medline: 28948205]

2. Safaeian M, Solomon D, Castle PE. Cervical cancer prevention--cervical screening: science in evolution. Obstet Gynecol Clin North Am 2007 Dec;34(4):739-760 [FREE Full text] [doi: 10.1016/j.ogc.2007.09.004] [Medline: 18061867]

3. Sahasrabuddhe VV, Parham GP, Mwanahamuntu MH, Vermund SH. Cervical cancer prevention in low- and middle-income countries: feasible, affordable, essential. Cancer Prev Res (Phila) 2012 Jan;5(1):11-17. [doi: 10.1158/1940-6207.CAPR-11-0540] [Medline: 22158053] 
4. Sankaranarayanan R, Anorlu R, Sangwa-Lugoma G, Denny LA. Infrastructure requirements for human papillomavirus vaccination and cervical cancer screening in sub-Saharan Africa. Vaccine 2013 Dec 29;31 Suppl 5:F47-F52. [doi: 10.1016/j.vaccine.2012.06.066] [Medline: 24331747]

5. Shanmugasundaram S, You J. Targeting persistent human papillomavirus infection. Viruses 2017 Aug 18;9(8):229 [FREE Full text] [doi: 10.3390/v9080229] [Medline: 28820433]

6. Bosch FX, Lorincz A, Muñoz N, Meijer CJLM, Shah KV. The causal relation between human papillomavirus and cervical cancer. J Clin Pathol 2002 Apr;55(4):244-265. [doi: 10.1136/jcp.55.4.244] [Medline: 11919208]

7. McGraw SL, Ferrante JM. Update on prevention and screening of cervical cancer. World J Clin Oncol 2014 Oct 10;5(4):744-752 [FREE Full text] [doi: 10.5306/wjco.v5.i4.744] [Medline: 25302174]

8. Koliopoulos G, Nyaga VN, Santesso N, Bryant A, Martin-Hirsch PP, Mustafa RA, et al. Cytology versus HPV testing for cervical cancer screening in the general population. Cochrane Database Syst Rev 2017 Aug 10;8:CD008587 [FREE Full text] [doi: 10.1002/14651858.CD008587.pub2] [Medline: 28796882]

9. Denny LA, Sankaranarayanan R, De Vuyst H, Kim JJ, Adefuye PO, Alemany L, et al. Recommendations for cervical cancer prevention in sub-saharan Africa. Vaccine 2013 Dec 29;31 Suppl 5:F73-F74. [doi: 10.1016/j.vaccine.2012.11.077] [Medline: $\underline{24331750]}$

10. Mohammed SI, Ren W, Flowers L, Rajwa B, Chibwesha CJ, Parham GP, et al. Point-of-care test for cervical cancer in LMICs. Oncotarget 2016 Apr 05;7(14):18787-18797 [FREE Full text] [doi: 10.18632/oncotarget.7709] [Medline: 26934314]

11. Goldie SJ, Gaffikin L, Goldhaber-Fiebert JD, Gordillo-Tobar A, Levin C, Mahé C, Alliance for Cervical Cancer Prevention Cost Working Group. Cost-effectiveness of cervical-cancer screening in five developing countries. N Engl J Med 2005 Nov 17;353(20):2158-2168. [doi: 10.1056/NEJMsa044278] [Medline: 16291985]

12. Barbee L, Kobetz E, Menard J, Cook N, Blanco J, Barton B, et al. Assessing the acceptability of self-sampling for HPV among Haitian immigrant women: CBPR in action. Cancer Causes Control 2010 Mar;21(3):421-431. [doi: 10.1007/s10552-009-9474-0] [Medline: 19943103]

13. Menard JM. The Social Context of Cervical Cancer Knowledge and Prevention Among Haitian Immigrant Women. 2008. URL: https://scholarcommons.usf.edu/etd/398/ [accessed 2020-09-01]

14. Petignat P, Faltin DL, Bruchim I, Tramèr MR, Franco EL, Coutlée F. Are self-collected samples comparable to physician-collected cervical specimens for human papillomavirus DNA testing? A systematic review and meta-analysis. Gynecol Oncol 2007 May;105(2):530-535. [doi: 10.1016/j.ygyno.2007.01.023] [Medline: 17335880]

15. Ogilvie GS, Patrick DM, Schulzer M, Sellors JW, Petric M, Chambers K, et al. Diagnostic accuracy of self collected vaginal specimens for human papillomavirus compared to clinician collected human papillomavirus specimens: a meta-analysis. Sex Transm Infect 2005 Jun;81(3):207-212. [doi: 10.1136/sti.2004.011858] [Medline: 15923286]

16. Szarewski A, Cadman L, Mallett S, Austin J, Londesborough P, Waller J, et al. Human papillomavirus testing by self-sampling: assessment of accuracy in an unsupervised clinical setting. J Med Screen 2007;14(1):34-42. [doi: 10.1258/096914107780154486] [Medline: 17362570]

17. Toliman PJ, Kaldor JM, Badman SG, Gabuzzi J, Silim S, Kumbia A, et al. Performance of clinical screening algorithms comprising point-of-care HPV-DNA testing using self-collected vaginal specimens, and visual inspection of the cervix with acetic acid, for the detection of underlying high-grade squamous intraepithelial lesions in Papua New Guinea. Papillomavirus Res 2018 Dec;6:70-76 [FREE Full text] [doi: 10.1016/j.pvr.2018.10.009] [Medline: 30391365]

18. Ogilvie G, Krajden M, Maginley J, Isaac-Renton J, Hislop G, Elwood-Martin R, et al. Feasibility of self-collection of specimens for human papillomavirus testing in hard-to-reach women. CMAJ 2007 Aug 28;177(5):480-483. [doi: 10.1503/cmaj.070013] [Medline: 17724326]

19. Institute of Medicine. Leveraging Culture to Address Health Inequalities: Examples from Native Communities: Workshop Summary. National Academies Press 2013 Dec 19. [doi: 10.17226/18496] [Medline: 24830070]

20. Liberati A, Altman DG, Tetzlaff J, Mulrow C, Gøtzsche PC, Ioannidis JPA, et al. The PRISMA statement for reporting systematic reviews and meta-analyses of studies that evaluate healthcare interventions: explanation and elaboration. BMJ 2009 Jul 21;339:b2700 [FREE Full text] [doi: 10.1136/bmj.b2700] [Medline: 19622552]

21. Aslam S, Emmanuel P. Formulating a researchable question: a critical step for facilitating good clinical research. Indian $\mathbf{J}$ Sex Transm Dis AIDS 2010 Jan;31(1):47-50 [FREE Full text] [doi: 10.4103/0253-7184.69003] [Medline: 21808439]

22. CASP Systematic Review Checklist. Critical Appraisal Skills Programme. URL: https://casp-uk.net/casp-tools-checklists/ [accessed 2020-09-22]

23. Katrak P, Bialocerkowski AE, Massy-Westropp N, Kumar VS, Grimmer KA. A systematic review of the content of critical appraisal tools. BMC Med Res Methodol 2004 Sep 16;4(1):22 [FREE Full text] [doi: 10.1186/1471-2288-4-22] [Medline: 15369598]

24. Thomas J, Harden A. Methods for the thematic synthesis of qualitative research in systematic reviews. BMC Med Res Methodol 2008 Jul 10;8(1):45 [FREE Full text] [doi: 10.1186/1471-2288-8-45] [Medline: 18616818]

25. Harden A, Garcia J, Oliver S, Rees R, Shepherd J, Brunton G, et al. Applying systematic review methods to studies of people's views: an example from public health research. J Epidemiol Community Health 2004 Sep 01;58(9):794-800. [doi: 10.1136/jech.2003.014829] [Medline: 15310807] 
26. Boland A, Cherry G, Dickson R, editors. Doing a Systematic Review: A Student's Guide, second edition. London: Sage Publishing; Oct 9, 2017.

27. Lee J, Carvallo M. Socioecological perspectives on cervical cancer and cervical cancer screening among Asian American women. J Community Health 2014 Oct;39(5):863-871. [doi: 10.1007/s10900-014-9887-x] [Medline: 24863746]

\title{
Abbreviations
}

CASP: Critical Appraisal Skills Programme

CINAHL: Cumulative Index to Nursing and Allied Health Literature

DNA: deoxyribonucleic acid

HPV: human papillomavirus

MeSH: Medical Subject Headings

PRISMA: Preferred Reporting Items for Systematic Reviews and Meta-Analyses

STI: sexually transmitted infection

\author{
Edited by G Eysenbach; submitted 05.06.20; peer-reviewed by J McElfresh, E Andrikopoulou; comments to author 29.07.20; revised \\ version received 11.08.20; accepted 18.08.20; published 22.10.20 \\ Please cite as: \\ Camara H, Zhang Y, Lafferty L, Vallely A, Guy R, Kelly-Hanku A \\ Qualitative Evidence Synthesis on Self-Collection for Human Papillomavirus-Based Cervical Screening: Protocol for Systematic \\ Review \\ JMIR Res Protoc 2020;9(10):e21093 \\ URL: https://www.researchprotocols.org/2020/10/e21093 \\ doi: $10.2196 / 21093$ \\ PMID: 33090114
}

CHawa Camara, Ye Zhang, Lise Lafferty, Andrew Vallely, Rebecca Guy, Angela Kelly-Hanku. Originally published in JMIR Research Protocols (http://www.researchprotocols.org), 22.10.2020. This is an open-access article distributed under the terms of the Creative Commons Attribution License (https://creativecommons.org/licenses/by/4.0/), which permits unrestricted use, distribution, and reproduction in any medium, provided the original work, first published in JMIR Research Protocols, is properly cited. The complete bibliographic information, a link to the original publication on http://www.researchprotocols.org, as well as this copyright and license information must be included. 